María Vidal Pascual, ${ }^{\dagger}$ Steffen Proemmel, ${ }^{\dagger}$ Winfried Beil,,

Rudolf Wartchow ${ }^{\S}$ and H. M. R. Hoffmann ${ }^{*},+$

\title{
Oxazatricyclic Noradamantanes: Stereocontrolled Synthesis of Functionalized Scopolines, Related Cage Molecules and Drug Leads
}

Spectroscopic data for $\mathbf{8 b}, 9 \mathrm{~b} \alpha, 9 \mathrm{~b} \alpha-\mathrm{Boc}, 10 \mathrm{~b}, 10-\mathrm{OH}, 11-\mathrm{OH}, 13-\mathrm{OH}$ and 11-NH-AlaCbz

$2 \alpha$-Benzyloxy-1,5-dimethyl-8-oxabicyclo[3.2.1 ] oct-6-en-3-one (8b): ${ }^{1} \mathbf{H}-\mathbf{N M R}\left(200 \mathrm{MHz}, \mathrm{CDCl}_{3}\right.$ mit TMS): $\delta_{\mathrm{H}}$ 7.41-7.29 (m, $\left.5 \mathrm{H}\right) ; 6.06\left(\mathrm{~d},{ }^{3} J=6 \mathrm{~Hz}, 1 \mathrm{H}\right) ; 6.00\left(\mathrm{~d},{ }^{3} J=6 \mathrm{~Hz}, 1 \mathrm{H}\right) ; 5.03\left(\mathrm{~d},{ }^{2} J=12 \mathrm{~Hz}, 1\right.$ $\mathrm{H}) ; 4.58\left(\mathrm{~d},{ }^{2} J=12 \mathrm{~Hz}, 1 \mathrm{H}\right) ; 3.81(\mathrm{~s}, 1 \mathrm{H}) ; 2.63\left(\mathrm{~d},{ }^{2} J=15 \mathrm{~Hz}, 1 \mathrm{H}\right) ; 2.44\left(\mathrm{dd},{ }^{2} J=15 \mathrm{~Hz}, \mathrm{~J} \leq 0.5 \mathrm{~Hz}, 1\right.$ $\mathrm{H}) ; 1.48$ (s, $6 \mathrm{H}) ;{ }^{13} \mathrm{C}-\mathrm{NMR}\left(50 \mathrm{MHz}, \mathrm{CDCl}_{3}\right.$ mit TMS): $\delta_{\mathrm{H}} 206.2(\mathrm{C}) ; 137.6(\mathrm{C}) ; 137.1 / 134.8(\mathrm{CH})$; $128.3(\mathrm{CH}) ; 127.9(\mathrm{CH}) ; 87.4(\mathrm{CH}) ; 86.7 / 84.8(\mathrm{C}) ; 74.4\left(\mathrm{CH}_{2}\right) ; 51.7\left(\mathrm{CH}_{2}\right) ; 23.1 / 20.5\left(\mathrm{CH}_{3}\right) ; \mathbf{I R}$ (KBr): 3034 w; 2976 m; 2929 m; 2874 m; 1718 vs; 1498 w; 1454 m; 1401 m; 1378 m; 1340 m; 1319 m; 1269 w; 1243 w; 1220 w; 1177 m; 1109 vs; 758 s; 705 s; Mass (RT): no $\mathrm{M}^{+}$: 167 (34.7, M+-Bn); 152 (29.8); 139 (14.0); 109 (19.4); 97 (20.0); 95 (15.2); 92 (11.8); 91 (100)

3 $\alpha$-Amino-2 $\alpha$-benzyloxy-1,5-dimethyl-8-oxabicyclo[3.2.1] oct-6-ene $\quad(\mathbf{9 b} \alpha):{ }^{1} \mathbf{H}-\mathbf{N M R} \quad(400 \mathrm{MHz}$, $\mathrm{CDCl}_{3}$ mit TMS): $\delta_{\mathrm{H}} 7.40-7.24(\mathrm{~m}, 5 \mathrm{H}) ; 6.38\left(\mathrm{~d},{ }^{3} J=5.8 \mathrm{~Hz}, 1 \mathrm{H}\right) ; 6.18\left(\mathrm{~d},{ }^{3} J=5.8 \mathrm{~Hz}, 1 \mathrm{H}\right) ; 4.86(\mathrm{~d}$, $\left.{ }^{2} J=11.7 \mathrm{~Hz}, 1 \mathrm{H}\right) ; 4.50\left(\mathrm{~d},{ }^{2} J=11.7 \mathrm{~Hz}, 1 \mathrm{H}\right) ; 3.87\left(\operatorname{tr},{ }^{3} J=6.2 \mathrm{~Hz}, 1 \mathrm{H}\right) ; 3.53\left(\mathrm{~d},{ }^{3} J=6.3 \mathrm{~Hz}, 1 \mathrm{H}\right)$; $2.33\left(\mathrm{~d},{ }^{2} J=15 \mathrm{~Hz}, 1 \mathrm{H}\right) ; 1.98\left(\mathrm{dd},{ }^{2} J=15 \mathrm{~Hz},{ }^{3} \mathrm{~J}=6 \mathrm{~Hz}, 1 \mathrm{H}\right) ; 1.35$ (s, $\left.3 \mathrm{H}\right) ; 1.33$ (s, $\left.3 \mathrm{H}\right) ;{ }^{13} \mathbf{C}-\mathbf{N M R}$ (100 MHz, $\left.\mathrm{CDCl}_{3}, \mathrm{TMS}\right): \delta_{\mathrm{H}} 139.8 / 135.3(\mathrm{CH}) ; 137.7(\mathrm{C}) ; 128.5(\mathrm{CH}) ; 128.3(\mathrm{CH}) ; 128.0(\mathrm{CH})$; 85.5/84.0 (C); $73.0\left(\mathrm{CH}_{2}\right) ; 70.4(\mathrm{CH}) ; 46.2(\mathrm{CH}) ; 35.4\left(\mathrm{CH}_{2}\right) ; 23.2 / 20.9\left(\mathrm{CH}_{3}\right)$; IR $(\mathrm{KBr}): 3251 \mathrm{~m}$; 3034 w; 2974 m; 2835 m; 1586 s; 1513 s, 1454 s; 1375 m; 1360 w; 1206 s; 1181 m; 1049 m; 1026 m; 895 m; Mass (rt): 259 (4.5, $\mathrm{M}^{+}$); 232 (1.6); 200 (1.3); 182 (3.3); 167 (18.5); 152 (19.6) 136 (9.3); 109 (20.2); 91 (100); 77 (10.9)

$3 \alpha$-Amino-(NH-tert-butyloxycarbonyl)-2 $\alpha$-benzyloxy-1,5-dimethyl-8-oxabicyclo[3.2.1]oct-6-ene (9bo-Boc): ${ }^{1}$ H-NMR (400 MHz, $\mathrm{CDCl}_{3}$, TMS): $\delta_{\mathrm{H}}$ 7.37-7.29 (m, $5 \mathrm{H}$ ); 6.15 (s, $2 \mathrm{H}$ ); 5.23 (d, ${ }^{3} J=9.3$ 
$\mathrm{Hz}, 1 \mathrm{H}) ; 4.58\left(\mathrm{~d},{ }^{2} J=11 \mathrm{~Hz}, 1 \mathrm{H}\right) ; 4.38\left(\mathrm{dtr},{ }^{3} J=6.6 \mathrm{~Hz},{ }^{3} J=6.7 \mathrm{~Hz}, 1 \mathrm{H}\right) ; 4.34\left(\mathrm{~d},{ }^{2} J=11 \mathrm{~Hz}, 1 \mathrm{H}\right)$; $3.59\left(\mathrm{~d},{ }^{3} J=6.7 \mathrm{~Hz}, 1 \mathrm{H}\right) ; 2.07\left(\mathrm{dd},{ }^{2} J=14.4 \mathrm{~Hz},{ }^{3} J=6.6 \mathrm{~Hz}, 1 \mathrm{H}\right) ; 1.83\left(\mathrm{~d},{ }^{2} J=14.4 \mathrm{~Hz}, 1 \mathrm{H}\right) ; 1.43$ (s, $9 \mathrm{H}) ; 1.41$ (s, $3 \mathrm{H}) ; 1.35$ (s, $3 \mathrm{H}) ;{ }^{13} \mathrm{C}-\mathrm{NMR}$ (100 MHz, CDCl 3 , TMS): $\delta_{\mathrm{H}} 155.6(\mathrm{C}) ; 138.0(\mathrm{C})$; 139.6/135.8 (CH); 128.3/128.1 (CH); $127.8(\mathrm{CH}) ; 85.7 / 84.4(\mathrm{C}) ; 79.1(\mathrm{C}) ; 78.7(\mathrm{CH}) ; 71.5\left(\mathrm{CH}_{2}\right)$; $44.9(\mathrm{CH}) ; 40.2\left(\mathrm{CH}_{2}\right) ; 28.4\left(\mathrm{CH}_{3}\right) ; 23.6\left(\mathrm{CH}_{3}\right) ; 21.1\left(\mathrm{CH}_{3}\right)$; IR (Golden Gate ATR): $3469 \mathrm{~m} ; 2976 \mathrm{~m}$; 2931 m; 2871 w; 1809 m; 1756 m; 1712 s; 1493 s; 1455 m; 1369 m; 1167 s; 1118 s; 1071 m; 950 m; Mass $\left(70^{\circ} \mathrm{C}\right): 359$ (0.8, $\left.\mathrm{M}^{+}\right) ; 303$ (4.2); 268 (1.9); 251 (2.4); 227 (5.7); 195 (10.1); 167 (12.5); 152 (31.5); 136 (32.6); 109 (30.0); 91 (100); 79 (8.2)

$7 \alpha$-Amino-(NH-tert-butyloxycarbonyl)-6 $\alpha$-benzyloxy-1,5-dimethyl-3,9-dioxatricyclo[3.3.1.0 $\left.{ }^{2,4}\right]$ nonane (10b): ${ }^{1} \mathrm{H}-\mathrm{NMR}\left(400 \mathrm{MHz}, \mathrm{CDCl}_{3}\right.$, TMS): $\delta_{\mathrm{H}} 7.30$ (m, $5 \mathrm{H}$ ); $5.03\left(\mathrm{dtr},{ }^{3} J=4.9 \mathrm{~Hz},{ }^{3} J=2.3 \mathrm{~Hz}, 1 \mathrm{H}\right.$ ); $4.57\left(\mathrm{~d},{ }^{2} J=11 \mathrm{~Hz}, 1 \mathrm{H}\right) ; 4.37\left(\mathrm{~d},{ }^{2} J=11 \mathrm{~Hz}, 1 \mathrm{H}\right) ; 3.55\left(\mathrm{~d},{ }^{3} J=2.8 \mathrm{~Hz}, 1 \mathrm{H}\right) ; 3.52\left(\mathrm{~d},{ }^{3} J=5 \mathrm{~Hz}, 1 \mathrm{H}\right)$; $3.41\left(\mathrm{~d},{ }^{3} J=2.8 \mathrm{~Hz}, 1 \mathrm{H}\right) ; 2.15\left(\mathrm{~d},{ }^{2} J=15.2 \mathrm{~Hz}, 1 \mathrm{H}\right) ; 1.92\left(\mathrm{dd},{ }^{2} J=15.1 \mathrm{~Hz},{ }^{3} J=5.6 \mathrm{~Hz}, 1 \mathrm{H}\right) ; 1.47$ (s, $9 \mathrm{H}) ; 1.39$ (s, $3 \mathrm{H}) ; 1.30$ (s, $3 \mathrm{H}) ;{ }^{13} \mathrm{C}-\mathbf{N M R}$ (100 MHz, CDCl 3 , TMS): $\delta_{\mathrm{C}} 155.9(\mathrm{C}) ; 137.0(\mathrm{C})$; 128.5/128.3 (CH); $127.8(\mathrm{CH}) ; 78.8 / 77.4(\mathrm{C}) ; 77.4(\mathrm{CH}) ; 76.7(\mathrm{C}) ; 71.7\left(\mathrm{CH}_{2}\right) ; 57.6 / 55.9(\mathrm{CH}) ; 44.8$ $(\mathrm{CH}) ; 38.7\left(\mathrm{CH}_{2}\right) ; 28.4\left(\mathrm{CH}_{3}\right) ; 19.9\left(\mathrm{CH}_{3}\right) ; 17.6\left(\mathrm{CH}_{3}\right)$; IR (Golden Gate ATR): $3430 \mathrm{w} ; 2976 \mathrm{~m} ; 2932$ m; 2871 w; 1709 vs; 1486 s; 1454 m; 1366 m; 1242 m; 1161 s; 1090 s; 1060 m; 957 m; Mass $\left(70^{\circ} \mathrm{C}\right)$ : 376 (1.4, M+1); 320 (9.7); 276 (6.5); 229 (3.3); 190 (9.6); 183 (11.7); 178 (10.4); 147 (21.3); 128 (8.4); 114 (10.7); 108 (21.7); 91 (100); 84 (62.9)

$7 \alpha$-Amino-(NH-tert-butyloxycarbonyl)-1,5-dimethyl-3,9-dioxatricyclo[3.3.1.0 $\left.0^{2,4}\right]$ nonan-6 $\alpha$-ol $(10-$ OH): ${ }^{1} \mathbf{H}-\mathbf{N M R}\left(400 \mathrm{MHz}, \mathrm{CDCl}_{3}, \mathrm{TMS}\right): \delta_{\mathrm{H}} 3.88\left(\mathrm{dtr},{ }^{3} \mathrm{~J}=6.15 \mathrm{~Hz},{ }^{3} J=2 \mathrm{~Hz}, 1 \mathrm{H}\right) ; 3.68\left(\mathrm{~d},{ }^{3} J=2.88\right.$ $\mathrm{Hz}, 1 \mathrm{H}) ; 3.67$ (s, $1 \mathrm{H}) ; 3.48\left(\mathrm{~d},{ }^{3} J=2.88 \mathrm{~Hz}, 1 \mathrm{H}\right) ; 3.22(\mathrm{~s}, 1 \mathrm{H}) ; 2.05\left(\mathrm{dd},{ }^{2} J=14.9 \mathrm{~Hz},{ }^{3} \mathrm{~J}=2 \mathrm{~Hz}, 1\right.$ $\mathrm{H}) ; 1.87$ (dd, $\left.{ }^{2} J=14.9 \mathrm{~Hz},{ }^{3} J=5.9 \mathrm{~Hz}, 1 \mathrm{H}\right) ; 1.47$ (s, $\left.9 \mathrm{H}\right) ; 1.33$ (s, $\left.3 \mathrm{H}\right) ; 1.25$ (s, $\left.3 \mathrm{H}\right) ;{ }^{3} \mathbf{C}-\mathbf{N M R}(100$ $\left.\mathrm{MHz}, \mathrm{CDCl}_{3}, \mathrm{TMS}\right): \delta_{\mathrm{C}} 158.4(\mathrm{C}) ; 80.8 / 78.5(\mathrm{C}) ; 74.5(\mathrm{C}) ; 72.2(\mathrm{CH}) ; 58.7 / 57.0(\mathrm{CH}) ; 49.7(\mathrm{CH}) ; 39.3$ $\left(\mathrm{CH}_{2}\right) ; 28.8\left(\mathrm{CH}_{3}\right) ; 20.1\left(\mathrm{CH}_{3}\right) ; 17.7\left(\mathrm{CH}_{3}\right)$; IR (Golden Gate ATR): $3412 \mathrm{~m} ; 2976 \mathrm{~m} ; 2933 \mathrm{~m} ; 1685 \mathrm{~s}$; 1487 s; 1454 m; 1366 s; 1243 m; 1160 s; 1084 s; 1048 m; 1022 m; 956 m; 863 m; Mass $\left(130{ }^{\circ} \mathrm{C}\right)$ : no $\mathrm{M}^{+} ; 228$ (5.3); 212 (6.1); 185 (2.1); 168 (22.4); 141 (39.0); 127 (52.3); 112 (73.3); 103 (56.9); 95 (37.4) 85 (72.4); $82(100)$

1,3-Dimethyl-2-oxa-6-aza-(N-tert-butyloxycarbonyl)-tricyclo[3.3.1.0 ${ }^{3,7}$ ]nonan-4 $\alpha, 8 \beta$-diol (11-OH): ${ }^{1}$ H-NMR (400 MHz, CDCl 3 , TMS): $\delta_{\mathrm{H}} 4.08$ (br s, $1 \mathrm{H}$ ); 3.94 (br s, $1 \mathrm{H} 7$ ); 3.87 (br s, $1 \mathrm{H}$ ); 3.68 (s, 1 $\mathrm{H}) ; 1.75\left(\mathrm{dd},{ }^{2} J=15.4 \mathrm{~Hz},{ }^{3} \mathrm{~J}=3.7 \mathrm{~Hz}, 1 \mathrm{H}\right) ; 1.47(\mathrm{~s}, 9 \mathrm{H}) ; 1.33(\mathrm{~s}, 3 \mathrm{H}) ; 1.29\left(\mathrm{~d},{ }^{2} J=15.6 \mathrm{~Hz}, 1 \mathrm{H}\right)$; 1.25 (s, $3 \mathrm{H}) ;{ }^{13} \mathrm{C}-\mathrm{NMR}\left(100 \mathrm{MHz}, \mathrm{CDCl}_{3}\right.$, TMS): $\delta_{\mathrm{C}} 155.5$ (C); 89.8/81.4 (C); $81.2(\mathrm{C}) ; 80.5(\mathrm{CH})$; $78.8(\mathrm{CH}) ; 69.1(\mathrm{CH}) ; 61.3(\mathrm{CH}) ; 40.5\left(\mathrm{CH}_{2}\right) ; 28.8\left(\mathrm{CH}_{3}\right) ; 22.3\left(\mathrm{CH}_{3}\right) ; 18.1\left(\mathrm{CH}_{3}\right)$; IR (Golden Gate ATR): 3427 m; 2980 m; 2935 m; 2909 w; 1682 vs; 1574 m; 1393 vs; 1368 m; 1258 m; 1164 vs; 1109 
m; 1091m; 1076 m; 1061m; 953 m; 856 m; Mass (90 C): 286 (3.8, $\left.\mathrm{M}^{+}+1\right) ; 229$ (8.4); 212 (3.5); 182 (6.6); 168 (9.3); 156 (100); 139 (77.2); 122 (13.6); 111 (32.2); 105 (21.7); 82 (12.1); 77 (16.1)

4及-Hydroxy-1,3-dimethyl-2-oxa-6-aza-(N-tert-butyloxycarbonyl)-tricyclo[3.3.1.0 ${ }^{3,7}$ ]nonan-8-one (13OH): ${ }^{1} \mathbf{H}-\mathrm{NMR}\left(400 \mathrm{MHz}, \mathrm{CDCl}_{3}, \mathrm{TMS}\right): \delta_{\mathrm{H}} 5.34\left(\mathrm{~d},{ }^{3} J=5.7 \mathrm{~Hz}, 1 \mathrm{H}\right) ; 4.47$ (br dd, ${ }^{3} J=7.4 \mathrm{~Hz},{ }^{3} J=7.2$ $\mathrm{Hz}, 1 \mathrm{H}) ; 3.56\left(\mathrm{~d},{ }^{3} J=2.8 \mathrm{~Hz}, 1 \mathrm{H}\right) ; 2.84\left(\mathrm{dd},{ }^{2} J=13.5 \mathrm{~Hz},{ }^{3} J=8 \mathrm{~Hz}, 1 \mathrm{H}\right) ; 1.89$ (br dd, ${ }^{2} J=13.4 \mathrm{~Hz}$, $\left.{ }^{2} J=11.2 \mathrm{~Hz}, 1 \mathrm{H}\right) ; 1.43$ (s, $\left.9 \mathrm{H}\right) ; 1.38$ (s, $\left.3 \mathrm{H}\right) ; 1.37$ (s, $\left.3 \mathrm{H}\right) ;{ }^{13} \mathbf{C}-\mathbf{N M R}\left(100 \mathrm{MHz}, \mathrm{CDCl}_{3}, \mathrm{TMS}\right): \delta_{\mathrm{C}}$ $200.9(\mathrm{C}) ; 155.6(\mathrm{C}) ; 82.8 / 78.4(\mathrm{C}) ; 80.3(\mathrm{C}) ; 78.6(\mathrm{CH}) ; 57.2(\mathrm{CH}) ; 56.7(\mathrm{CH}) ; 45.6\left(\mathrm{CH}_{2}\right) ; 28.3$ $\left(\mathrm{CH}_{3}\right) ; 19.1\left(\mathrm{CH}_{3}\right) ; 13.3\left(\mathrm{CH}_{3}\right)$; IR (Golden Gate ATR): 3368 m; 2976 m; 2930 m; 2909 w; 1779 w; 1694 vs; 1451 m; 1392 m; 1367 s; 1245 m; 1163 vs; 1099 vs; 956 m; 863 m; Mass (90C): 244 (14.1, no $\mathrm{M}^{+}$); 243 (33.6); 226 (16.2); 183 (100); 168 (17.4); 156 (34.2); 139 (56.3); 128 (71.1); 114 (74.7); $94(70.5) ; 82(96.4)$

8 $\alpha$-Amino-(N-Z-alanine)-1,3-dimethyl-2-oxa-6-aza-(N-tert-butyloxycarbonyl)-tricyclo[3.3.1.0

${ }^{3,7}$ Jnonan-4 $\alpha$-ol (11-NH-AlaCbz): ${ }^{1} \mathbf{H}-\mathbf{N M R}\left(400 \mathrm{MHz}, \mathrm{CDCl}_{3}\right.$, TMS): $\delta_{\mathrm{H}} 7.38-7.26$ (m, $\left.5 \mathrm{H}\right) ; 5.19$ (d, $\left.{ }^{3} J=3.8 \mathrm{~Hz}, 1 \mathrm{H}\right) ; 5.14\left(\mathrm{~d},{ }^{2} J=12 \mathrm{~Hz}, 1 \mathrm{H}\right) ; 5.05\left(\mathrm{~d},{ }^{2} J=12 \mathrm{~Hz}, 1 \mathrm{H}\right) ; 4.72(\mathrm{~s}, 1 \mathrm{H}) ; 4.51\left(\mathrm{q},{ }^{3} J=7 \mathrm{~Hz}, 1\right.$ $\mathrm{H}) ; 4.47\left(\operatorname{tr},{ }^{3} \mathrm{~J}=7.3 \mathrm{~Hz}, 1 \mathrm{H}\right) ; 3.56\left(\mathrm{~d},{ }^{3} \mathrm{~J}=2.8 \mathrm{~Hz}, 1 \mathrm{H}\right) ; 1.75\left(\mathrm{dd},{ }^{2} J=15.4 \mathrm{~Hz},{ }^{3} \mathrm{~J}=3.7 \mathrm{~Hz}, 1 \mathrm{H}\right) ; 1.45$ (s, $9 \mathrm{H}) ; 1.36\left(\mathrm{~d},{ }^{3} \mathrm{~J}=7 \mathrm{~Hz}, 3 \mathrm{H} ; 1.34\right.$ (s, $\left.3 \mathrm{H}\right) ; 1.28$ (d, $\left.{ }^{2} \mathrm{~J}=15.6 \mathrm{~Hz}, 1 \mathrm{H}\right) ; 1.25$ (s, $\left.3 \mathrm{H}\right) ;{ }^{13} \mathbf{C}-\mathbf{N M R}(100$ $\left.\mathrm{MHz}, \mathrm{CDCl}_{3}, \mathrm{TMS}\right): \delta_{\mathrm{C}} 156.5(\mathrm{C}) ; 155.6(\mathrm{C}) ; 136.3(\mathrm{C}) ; 128.7 / 128.6(\mathrm{CH}) ; 128.2(\mathrm{CH}) ; 83.8 / 78.3$ (C5); $80.8(\mathrm{C}) ; 77.3(\mathrm{CH}) ; 65.4(\mathrm{CH}) ; 54.8(\mathrm{CH}) ; 50.3(\mathrm{CH}) ; 41.5\left(\mathrm{CH}_{2}\right) ; 27.9\left(\mathrm{CH}_{3}\right) ; 20.7\left(\mathrm{CH}_{3}\right) ; 18.7$ $\left(\mathrm{CH}_{3}\right) ; 14.8\left(\mathrm{CH}_{3}\right)$; IR (Golden Gate ATR): 3323 m; 3033 w; 2932 m; 2855 m; 1748 m; 1703 vs; 1662 s; 1524 s; 1454 s; 1389 w; 1339 m; 1256 s; 1213 s; 1068 s; 1028 m; 966 m; MS-FAB: $489\left(3\right.$, M$^{+}$); 463 (5); 391 (15); 305 (100); 287 (22); 261 (47); 243 (24); 227 (29); 215 (55) 